University of Nebraska - Lincoln

DigitalCommons@University of Nebraska - Lincoln

4-30-2001

\title{
Asymmetric nanoscale switching in ferroelectric thin films by scanning force microscopy
}

Alexei Gruverman

University of Nebraska-Lincoln, agruverman2@unl.edu

A. Kholkin

University of Aveiro, kholkin@cv.ua.pt

A. I. Kingon

North Carolina State University, Raleigh, angus_kingon@brown.edu

H. Tokumoto

Joint Research Center for Atom Technology-National Institute for Advanced Interdisciplinary Research (JRCAT-NAIR), Tsukuba, Ibaraki 305, Japan

Follow this and additional works at: https://digitalcommons.unl.edu/physicsgruverman

Part of the Physics Commons

Gruverman, Alexei; Kholkin, A.; Kingon, A. I.; and Tokumoto, H., "Asymmetric nanoscale switching in ferroelectric thin films by scanning force microscopy" (2001). Alexei Gruverman Publications. 7. https://digitalcommons.unl.edu/physicsgruverman/7

This Article is brought to you for free and open access by the Research Papers in Physics and Astronomy at DigitalCommons@University of Nebraska - Lincoln. It has been accepted for inclusion in Alexei Gruverman Publications by an authorized administrator of DigitalCommons@University of Nebraska - Lincoln. 


\title{
Asymmetric nanoscale switching in ferroelectric thin films by scanning force microscopy
}

\author{
A. Gruverman ${ }^{\text {a) }}$ \\ Department of Materials Science and Engineering, North Carolina State University, Raleigh, \\ North Carolina 27695
}

A. Kholkin

Department of Ceramics and Glass Engineering, University of Aveiro, 3810-193 Aveiro, Portugal

A. Kingon

Department of Materials Science and Engineering, North Carolina State University, Raleigh, North Carolina 27695

\section{H. Tokumoto}

Joint Research Center for Atom Technology_National Institute for Advanced Interdisciplinary Research (JRCAT-NAIR), Tsukuba, Ibaraki 305, Japan

(Received 13 November 2000; accepted for publication 21 February 2001)

\begin{abstract}
Scanning force microscopy (SFM) has been used to perform nanoscale studies of the switching behavior of $\mathrm{Pb}(\mathrm{Zr}, \mathrm{Ti}) \mathrm{O}_{3}$ thin films via the direct observation of their domain structures. The study revealed a significant asymmetry of a switching pattern which is a function of the voltage polarity and original domain structure of individual grains. The phenomenon of asymmetric switching is attributed (1) to the presence of an internal built-in electric field at the bottom interface and (2) to the mechanical stress exerted by the SFM tip. The former effect results in incomplete $180^{\circ}$ switching, while the latter effect leads to a $90^{\circ}$ rotation of the polarization vector. The resulting shear stress deformation of the grain underneath the tip combined with the applied field effect propels polarization reversal in the adjacent grains. (C) 2001 American Institute of Physics.
\end{abstract}

[DOI: $10.1063 / 1.1366644]$

Ferroelectric thin films possess a unique set of physical properties which make them promising materials for application in various electronic devices. ${ }^{1}$ For successful implementation of ferroelectric devices based on polarization reversal, for example nonvolatile random access memories, symmetric switching between two opposite polarization states must be ensured. However, it is often observed that ferroelectric films exhibit significant imprint, or asymmetry of switching parameters, such as the coercive voltage and the remanent polarization. ${ }^{2-7}$ This effect may lead to a write failure when the ferroelectric capacitor cannot be switched by the programming voltage due to an increase in the coercive voltage at a certain polarization state. The cause of the voltage shift is generally attributed to the presence of an internal electric field which supports the given polarization state while opposing the antiparallel one. However, there is a lack of understanding of the microscopic mechanism of asymmetric behavior of ferroelectric capacitors. The necessity of scaling the size of a storage element in high-density ferroelectric memories down to the submicrometer range would seem to require a detailed investigation of the mechanism of asymmetric switching at the nanoscale level.

Recently, significant progress made in application of scanning force microscopy (SFM) to the characterization of ferroelectric materials has provided a unique opportunity to obtain microscopic information on switching processes in ferroelectric thin films by direct observation of their domain

${ }^{a)}$ Electronic mail: alexei_gruverman@ncsu.edu structures. ${ }^{8,9}$ It has been shown that nanosize ferroelectric domains can be successfully written and detected by means of piezoresponse SFM. ${ }^{9-12}$ It should be noted that, due to the absence of a top electrode in piezoresponse studies, the tip/ film/electrode heterostructure brings about its own asymmetry which may complicate the nanoscale investigation and interpretation of the mechanism of "intrinsic" switching asymmetry and imprint in ferroelectric films. However, SFM observation of domain evolution during polarization reversal can help to better understand the origin of asymmetric switching in ferroelectric capacitors. The purpose of the present letter is to investigate the process of polarization reversal at the nanoscale level and to assess the effect of the as-grown domain pattern and voltage polarity on the switching behavior of ferroelectric $\mathrm{Pb}(\mathrm{Zr}, \mathrm{Ti}) \mathrm{O}_{3}$ (PZT) films.

The SFM piezoresponse imaging method, used in this study, is described in detail elsewhere. ${ }^{8,9}$ A conductive gold coated $\mathrm{Si}_{3} \mathrm{~N}_{4}$ cantilever was used both for domain visualization and for polarization reversal. The domains were imaged by scanning the film with an applied ac $3 \mathrm{~V}$ voltage (peakto-peak) oscillating at $10 \mathrm{kHz}$. Experiments were carried out using $210 \mathrm{~nm}$ polycrystalline $\mathrm{Pb}\left(\mathrm{Zr}_{0.2} \mathrm{Ti}_{0.8}\right) \mathrm{O}_{3}$ films with $\mathrm{La}_{0.5} \mathrm{Sr}_{0.5} \mathrm{CoO}_{3}$ (LSCO) bottom electrodes deposited on Pt/ $\mathrm{TiN} / \mathrm{Si}$ substrates.

Switching voltage pulses were applied through the probing tip positioned at the centers of single grains which exhibited sharp as-grown domain contrast. Under the present experimental conditions domains with the polarization vector oriented toward the bottom electrode (hereafter designated "positive" domains) appeared as dark regions, while do- 

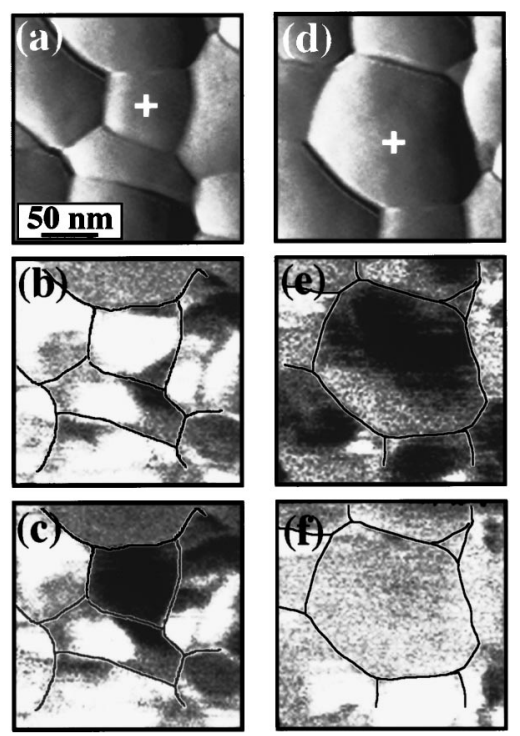

FIG. 1. Topographic (a), (d) and piezoresponse (b), (c), (e), (f) images of the PZT film illustrating asymmetry of switching for negative, oriented upward, and positive, oriented downward, domains; (b), (e) original as-grown domain structures with negatively (b) and positively (e) polarized grains in the center; (c), (f) domain structures after $3 \mathrm{~V}$ (c) and $-3 \mathrm{~V}$ (f) voltage pulse application. White crosses in (a) and (d) indicate the tip position during voltage pulse application.

mains oriented upward ("negative" domains) emerged as bright regions. Figure 1 shows the results of voltage application $(3 \mathrm{~V}, 1 \mathrm{~s})$ to two single grains which were originally polarized in opposite directions. It can be seen that the negatively polarized grain exhibits an inverse contrast after voltage application [Figs. 1(b) and 1(c)] suggesting $180^{\circ}$ polarization reversal. It should be noted that the switching is confined within the grain boundaries. On the other hand, application of an opposite switching voltage of the same amplitude to the positively polarized grain did not result in an inverse contrast [Figs. 1(e) and 1(f)]: the black grain becomes gray. At the same time we observe that polarization reversal has occurred in the adjacent grains as indicated by the inverse domain contrast of the grains in the lower part of Fig. 1(f). The gray contrast in this case can be attributed to the incomplete $180^{\circ}$ switching and/or the formation of $90^{\circ}$ domains. 8,9

There could be two alternative explanations for the observed asymmetry of switching. First, it can be attributed to the presence of an internal built-in electric field $E_{\mathrm{bi}}$ in the film. Such a built-in field could be related, for example, to the Schottky barrier between a ferroelectric with semiconductor properties and a metal bottom electrode. ${ }^{7,13}$ This field, which exists in the vicinity of the film/electrode interface, is not polarization dependent and is always pointing in one direction. It has been shown by interferometric studies of the self-polarization effect in PZT films ${ }^{7}$ that the internal built-in field could reach a value of the order of $10^{5} \mathrm{kV} / \mathrm{cm}$. This field is sufficiently high to polarize a part of the film adjacent to the bottom electrode. It is thus possible that the effect of asymmetric switching shown in Figs. 1(e) and 1(f) is due to the inability of the electric field applied between the tip and the bottom electrode to reverse the polarization in the entire film beneath the tip. When the external field is applied parallel to $E_{\text {bi }}$, full switching occurs as shown in Figs. 1(b) and

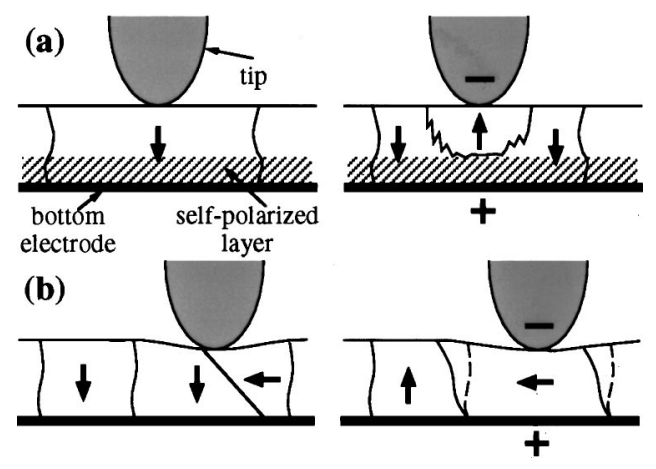

FIG. 2. (a) Diagram illustrating incomplete $180^{\circ}$ switching of a positive domain under a negative voltage applied to the tip and formation of counter domains due to the presence of the built-in internal field near the bottom electrode. A self-polarized region with the built-in field pointing to the electrode is shown by the shadowed area. (b) Diagram illustrating $90^{\circ}$ rotation of the polarization vector in the grain underneath the tip due to compressive stress exerted by the tip. Note shear stress gradient assisted $180^{\circ}$ switching in the adjacent grain.

1(c). In contrast, when the applied field is antiparallel to the internal field, polarization reversal occurs only in the upper part of the grain which results in the formation of a nonthrough counter domain and leads to the gray contrast of the grain in the piezoresponse image [Fig. 2(a)]. Based on the observed asymmetry of the switching behavior, we conclude that the $E_{\mathrm{bi}}$ field at the PZT/LSCO interface points towards the electrode. Even a small built-in field at the bottom electrode can prevent polarization reversal in the lower part of the film given that the field generated by the SFM tip decreases quadratically in the polar direction. ${ }^{9}$ It should be noted that the effect observed in Figs. 1(e) and 1(f) is not $100 \%$ reproducible: sometimes, when the whole grain is uniformly polarized, $180^{\circ}$ switching occurs easily for positive domains. This differing behavior can be due to statistical variations in the Schottky barrier height from grain to grain. This observation is consistent with the fact that the PZT/ LSCO film is in the polydomain state after deposition.

The second explanation of asymmetrical switching involves consideration of the original domain structure of a grain and the mechanical stress exerted on the grain by the probing tip. It can be seen from Figs. 1(d) and 1(e) that the poling voltage was applied near the border between the gray and black regions which, in principle, could be interpreted as a $90^{\circ}$ domain boundary. When the tip contacts the surface during the poling experiment, it exerts compressive mechanical stress in the direction normal to the film surface. This is schematically shown in Fig. 2(b). This stress can be estimated by dividing the loading (contact) force between the probing tip and the film (a typical setting in our SFM piezoelectric measurements is about $10^{-8} \mathrm{~N}$ ) by the effective tipsample contact area $\left(\sim 100 \mathrm{~nm}^{2}\right)$. The resulting compressive stress is about $100 \mathrm{MPa}$ and is high enough to produce a noticeable domain rearrangement as has been well documented in bulk PZT. ${ }^{14}$ On one hand, due to compressive stress and mechanical clamping of the grain underneath the tip, $180^{\circ}$ polarization switching in this area may be hampered. (This may explain the so-called "doughnut" effect observed in piezoresponse $\mathrm{SFM}^{15}$ when a grain area right underneath the probing tip remained unswitched after voltage application while a surrounding area was fully switched.) 
On the other hand, due to the high value of the dielectric constant of PZT films, the external electric field should have a component parallel to the film surface outside of the tipsample contact area. ${ }^{16}$ Thus, the combined action of the applied electric field and mechanical stress may result in a $90^{\circ}$ rotation of the polarization vector, so that the $90^{\circ}$-domain wall will be moved until the entire grain becomes $a$-oriented, i.e., with an in-plane polarization [Fig. 2(b)]. As a result, the grain will appear with gray contrast in the piezoresponse image. It should be noted that it is the presence of $90^{\circ}$ or non- $180^{\circ}$ domains in the grains that enhances the possibility of this scenario.

At the same time, this scenario should lead to shear deformation of the grain [Fig. 2(b)] which could help to understand why polarization reversal occurred in the adjacent grains [Fig. 1(f)]. This deformation can be estimated as: $\Delta l / l=d_{15} E$, where $d_{15}$ is the shear piezoelectric coefficient, $E$ is the normal component of the external field, and $\Delta l$ is the lateral displacement of the area with characteristic length $l$ in contact with the tip. The shear piezoelectric coefficient is much higher than both the $d_{33}$ and $d_{31}$ coefficients and can be as high as $500 \mathrm{pm} / \mathrm{V}$ for PZT ceramics. ${ }^{14} \mathrm{In}$ a film with dense grain structure, the shear deformation will be transferred to the adjacent grains causing a stress gradient which can be considered as an additional source of the internal electric field and can facilitate domain reorientation in these grains. ${ }^{17}$ We believe that the mechanical stress gradient itself is not enough to cause polarization reversal in the adjacent grains. On our opinion, it is due to the combination of the shear stress gradient and the long-range character of the tipgenerated external field ${ }^{8}$ that the $180^{\circ}$ switching occurs in the adjacent grains. (It has been shown in earlier publications $^{8}$ that the size of a $180^{\circ}$ domain written in SFM can be as large as $1 \mu \mathrm{m}$ in diameter.) As a result, the polarization can be switched in the adjacent grains while in the central grain, clamped by the tip, the switching will be incomplete. Obviously, the shear stress effect will result in opposite stress gradients in the grains neighboring the "tail" or "head"' of the $90^{\circ}$ domain in the central grain and, therefore, not all adjacent grains will be switched. It can be seen that only the grains in the bottom part of Fig. 1(f) are reversed which is in qualitative agreement with the proposed model. Clear distinction between the two described mechanisms could be made with the help of complementary inplane polarization measurements. ${ }^{12}$

The effect of the original domain structure on the switching pattern and the possibility of polarization vector rotation under the compressive stress of the SFM tip are clearly illustrated in Fig. 3. Application of the switching voltage of $3 \mathrm{~V}$ to a grain in a polydomain state [Figs. 3(a) and $3(b)$ ] resulted in the formation of a group of lamellar (probably $90^{\circ}$ ) domains [Fig. 3(c)] which is a strong indication of non- $180^{\circ}$ switching. Further examination revealed that these domains spontaneously moved toward the grain edge apparently due to relaxation of the mechanical stress induced during switching [Fig. 3(d)].

Thus, the asymmetric nanoscale switching in SFM can be attributed to two effects: (1) the effectively "unswitchable" polarization in the vicinity of the bottom electrode due to the presence of the internal built-in electric field, and (2)

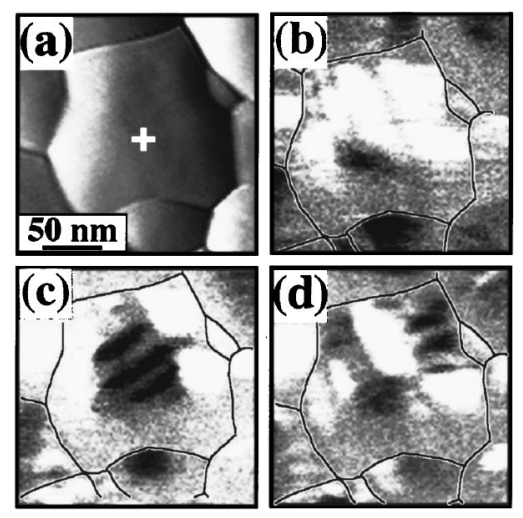

FIG. 3. Formation of lamellar domains as a result of non- $180^{\circ}$ switching. Topographic (a) and piezoresponse (b)-(d) images of the PZT film; (b) original domain structure; (c) domain structure immediately after voltage pulse application; (d) domain structure 15 min after voltage pulse application.

the mechanical stress exerted by the probing tip. The latter effect, which becomes particularly important in the case of switching of polydomain structures containing $90^{\circ}$ domains, causes, via the shear stress gradient, polarization reversal in grains far beyond the tip-film contact area. Obviously, further work is required to separate the tip-induced effects from the "true" switching which occurs in a typical thin film capacitor with a low aspect ratio. On the other hand, both electrical and mechanical mechanisms take place in reality. Therefore, the obtained SFM data, which revealed significant effect of asymmetric field and mechanical coupling between grains, could be useful for evaluation of the switching behavior of nanoscale ferroelectric capacitors with constrained geometry, where fringing fields and mechanical stresses could severely limit their functionality.

This work was supported by DARPA (Grant No. N39998-98-C-3566) and NEDO, Japan.

${ }^{1}$ O. Auciello, J. F. Scott, and R. Ramesh, Phys. Today 51, 22 (1998).

${ }^{2}$ W. L. Warren, D. Dimos, G. E. Pike, B. A. Tuttle, M. V. Raymond, R. Ramesh, and J. T. Evans, Appl. Phys. Lett. 67, 866 (1995).

${ }^{3}$ J. Lee, C. H. Choi, B. H. Park, T. W. Noh, and J. K. Lee, Appl. Phys. Lett. 72, 3380 (1998).

${ }^{4}$ T. Fukami and S. Fujii, Jpn. J. Appl. Phys., Part 1 24, 632 (1985).

${ }^{5}$ S. Sun, L. Liu, and P. A. Fuierer, Ferroelectrics 152, 187 (1994).

${ }^{6}$ R. Bruchhaus, D. Pitzer, R. Primig, W. Wersing, and Y. Xu, Integr. Ferroelectr. 14, 141 (1997).

${ }^{7}$ A. Kholkin, K. Brooks, D. Taylor, S. Hiboux, and N. Setter, Integr. Ferroelectr. 22, 525 (1998).

${ }^{8}$ A. Gruverman, O. Auciello, and H. Tokumoto, Integr. Ferroelectr. 19, 49 (1998).

${ }^{9}$ A. Gruverman, O. Auciello, and H. Tokumoto, Annu. Rev. Mater. Sci. 28, 101 (1998).

${ }^{10}$ T. Hidaka, T. Maruyama, I. Sakai, M. Saitoh, L. A. Wills, R. Hiskes, S. A. Dicarolis, and J. Amano, Integr. Ferroelectr. 17, 319 (1997);

${ }^{11}$ C. Harnagea, A. Pignolet, M. Alexe, K. M. Satyalakshmi, D. Hesse, and U. Goesele, Jpn. J. Appl. Phys., Part 2 38, L1255 (1999).

${ }^{12}$ A. Roelos, U. Bottger, R. Waser, F. Schlaphof, S. Trogisch, and L. Eng, Appl. Phys. Lett. 77, 3444 (2000).

${ }^{13}$ J. F. Scott, Integr. Ferroelectr. 9, 1 (1995).

${ }^{14}$ B. Jaffe, W. R. Cook, and H. Jaffe, Piezoelectric Ceramics (R.A.N. Marietta, OH, 1971).

${ }^{15}$ A. Gruverman (unpublished); M. Alexe (private communication).

${ }^{16}$ B. I. Bleaney and B. Bleaney, Electricity and Magnetism (Clarendon, Oxford, 1965), p. 57.

${ }^{17}$ K. Abe and S. Komatsu, J. Appl. Phys. 77, 6461 (1995). 